\title{
Fatores demográficos e socioeconômicos associados à cárie dentária em uma comunidade nordestina de baixa renda
}

\section{Demographic and socioeconomic factors associated with dental caries in a low-income Northeastern community}

\author{
Jordania Rodrigues Landim* \\ Maria Cleuba Araújo Freire Neta* \\ Mychele Cavalcante de Andrade Martins* \\ Sharmênia de Araújo Soares Nuto* \\ José Ueleres Braga ${ }^{* * *}$
}

\section{Resumo}

Objetivo: esta pesquisa tem como objetivo estudar a relação entre condições socioeconômicas e demográficas, com cárie e suas sequelas, em indivíduos adultos e idosos que vivem em uma comunidade nordestina. Métodos: foram aplicados questionários para caracterização domiciliar e da condição socioeconômica, realizando-se, além disso, exames bucais em 141 indivíduos, tendo 83 (59\%) entre 35 a 44 anos e 58 (41\%) acima de 65 anos. A análise dos dados consistiu no cálculo das médias, intervalos de confiança de 95\%, medianas, valores mínimos e máximos. Para testar hipóteses sobre a associação do uso e da necessidade de próteses com grupo etário, sexo, classe econômica e raça/etnia, foi calculado o qui-quadrado. O CPOD (índice de dentes cariados, perdidos e obturados) foi dicotomizado entre alto e baixo para o cálculo do risco relativo. O nível de significância estatística usado foi de 5\%. Resultados: Na análise do CPOD, não se obteve diferença significativa na característica sexo, onde ambos tiveram uma prevalência alta. A população negra estudada apresentou uma média de dentes cariados menor que as raças branca e indígena, com valores de $3,39,4,60$ e 6,50, respectivamente. As mulheres usam mais prótese superior quando comparadas aos homens ( $p=0,016)$. Eles, por sua vez, têm maior necessidade de utilização dessa prótese $(p=0,008)$. Conclusão: este estudo permitiu concluir que sexo, classe econômica e raça/cor não influenciaram o CPOD.

Palavras-chave: Saúde bucal. Levantamentos epidemiológicos. Cárie dentária. Condições sociais.

\section{Introdução}

As desigualdades socioeconômicas e seu impacto nas condições de saúde das pessoas e dos grupos humanos estão sendo temas bastante discutidos em saúde coletiva. A relação existente entre as condições sociais e econômicas e os diferenciais de risco para muitas doenças, bem como de acesso a serviços de saúde, coloca em foco o conceito de Determinantes Sociais de Saúde (DSS) para que seja possível identificar e eliminar os fatores prejudiciais à saúde da população ${ }^{1}$.

A Comissão Nacional sobre os Determinantes Sociais da Saúde (CNDSS) define os DSS como a influência dos fatores sociais, econômicos, culturais, étnicos/raciais, psicológicos e comportamentais na ocorrência de problemas de saúde e seus fatores de risco na população. A comissão homônima da Organização Mundial de Saúde (OMS), por sua vez, adota uma definição mais curta, sendo os DSS a relação direta das condições sociais com o modo de viver e trabalhar ${ }^{2}$.

Os estudos sobre as relações entre determinantes sociais em saúde apresentam alguns desafios, como relacionar hierarquicamente os fatores mais gerais associados à saúde (sociais, econômicos, políticos) e as mediações de como tais fatores influenciam a situação de saúde de pessoas e grupos, já que

\footnotetext{
Cirurgiã-dentista, curso de Odontologia, Universidade de Fortaleza (Unifor), Fortaleza, Ceará, CE, Brasil.

Doutora em Ciências da Saúde, curso de Odontologia, Universidade de Fortaleza (Unifor), Fortaleza, CE, Brasil.

Doutor em Epidemiologia, curso de Medicina, Universidade Estadual do Rio de Janeiro (UERJ), Rio de Janeiro, RJ, Brasil.
} 
essa relação não se constitui, diretamente, como causa-efeito. Outro desafio importante a ser destacado refere-se à diferenciação dos determinantes de saúde que atingem um grupo de pessoas ou um único indivíduo isoladamente, pois os fatores que influenciam a saúde em âmbito individual não são os mesmos a influenciar coletivamente ${ }^{3,4}$, na medida em que, para a epidemiologia social, as causas do adoecimento da sociedade devem ser priorizadas em relação às causas individuais ${ }^{5}$.

O estudo das condições sociais como produtora de doenças bucais ainda se encontra em fase inicial $^{6,1}$. Apesar disso, o número de pesquisas sobre a determinação social em saúde dentro da Odontologia cresceu nas duas últimas décadas, destacando as perdas dentárias como principal marca da desigualdade social, sendo a cárie dental e a doença periodontal as principais responsáveis pela perda dos elementos dentários ${ }^{7}$.

O planejamento de políticas preventivas e assistenciais em saúde bucal no Brasil ainda não é dos mais adequados, está "engatinhando" no âmbito do atendimento universal e igualitário. Isso se deve tanto aos estudos epidemiológicos em saúde bucal, que, por muito tempo, concentraram-se exclusivamente na população infantil, particularmente em escolares, resultando em poucos estudos investigativos de agravos e doenças bucais em adultos e idosos ${ }^{8}$, quanto ao fato de que esses mesmos grupos não eram tratados como áreas de atenção prioritária, resultando em altos índices de edentulismo e alta prevalência de cárie e doença periodontal ${ }^{9}$.

De acordo com Fure e Zickert ${ }^{10}$ (1997), a perda dentária é atribuída, principalmente, à cárie dentária, incluindo cáries radiculares. Apesar da diminuição do índice CPO-D no Brasil como um todo, existe, ainda, uma grande iniquidade em sua distribuição, e uma das explicações consiste nas condições degradantes de vida a que uma considerável parcela da população é submetida ${ }^{11}$.

Assim, esta pesquisa tem como objetivo estudar a relação entre condições socioeconômicas e demográficas com cárie e suas sequelas em indivíduos adultos e idosos que vivem em uma comunidade nordestina de baixa renda, a fim de subsidiar o desenvolvimento de programas direcionados a populações semelhantes.

\section{Métodos}

Trata-se de um estudo transversal com abordagem descritiva e analítica. Realizou-se na Comunidade do Dendê e cercanias, local onde a população participante deste trabalho habita, situada no Bairro Edson Queiroz, no município de Fortaleza-CE. Para demarcar e conhecer a população de um amplo projeto sobre a saúde dessa comunidade, no período de outubro de 2007 a abril de 2008, foi realizado um cadastramento do número de habitantes e, também, o georreferenciamento das residências da área, denominada de fase zero da pesquisa, quando foram cadastrados 3.717 domicílios e 10.892 moradores $^{12}$.

A amostra desse estudo inclui indivíduos de dois grupos: o primeiro, de adultos com idades entre 35 e 44 anos; e o segundo, de idosos acima de 65 anos de idade, de ambos os sexos. Visitas a esses moradores específicas para esse estudo ocorreram no período de janeiro a outubro de 2010. A amostra foi composta por 5,8\% dos moradores adultos, correspondendo a 83 examinados, e 18,4\% dos idosos cadastrados na fase zero, ou seja, 58 indivíduos. O cálculo da amostra deste estudo baseou-se na fase linha de base, e a amostra foi selecionada de forma aleatória simples, realizada por meio do aplicativo estatístico STATA 9.0 ${ }^{\circledR 13}$. Dentro da amostra do estudo, existiram recusas dos participantes, não localização dos indivíduos ou de seus domicílios, além dos domicílios demolidos ou fechados. O alto percentual de domicílios não visitados $(23,0 \%)$ pode ser devido tanto aos endereços incompletos como à rapidez dos deslocamentos urbanos ${ }^{14}$.

Os desfechos deste estudo são os diagnósticos e níveis de gravidade da cárie dentária, utilizando os índices: CPO-D (13), CPOD presente, CPOD passado, uso e necessidade de prótese ${ }^{15}$.

O CPO-D é o índice mais usado em levantamentos epidemiológicos na Odontologia, recomendado pela $\mathrm{OMS}^{16}$ para medir e comparar a experiência de cárie dentária em populações; seu valor expressa a média de dentes cariados $(\mathrm{C})$, perdidos $(\mathrm{P})$ e obturados $(\mathrm{O})$ em um grupo de indivíduos ${ }^{7}$. Quando observados no indivíduo os componentes cariados e/ ou extração indicada, constata-se a doença presente (CPOD presente), enquanto a presença de dentes restaurados e/ou perdidos por cárie indica as sequelas da doença passada (COPD passado). Neste estudo, buscou-se utilizar não somente o CPOD, mas também essa subdivisão entre doença presente ou doença passada, pois tal informação é importante para identificar a necessidade de acesso aos serviços odontológicos, principalmente na população adulta e idosa.

As perguntas do questionário demográfico e socioeconômico referiam-se a idade, sexo, raça ou cor dos indivíduos e sua classe econômica. A idade em anos foi comprovada pela data de nascimento dos participantes; o sexo, segundo a classificação de gênero masculino ou feminino. A característica raça/ cor foi considerada com base na autodeclaração do indivíduo, sendo possíveis as seguintes classificações: amarelo, branco, indígena, negro e pardo ${ }^{15}$. A classe econômica foi obtida de acordo com o "Critério de Classificação Econômica Brasil”, que utiliza o levantamento de características domiciliares (presença e quantidade de alguns itens domiciliares de conforto e grau de escolaridade do chefe de família) para diferenciar a população, abandonando 
a pretensão de classificar a população em termos de "classes sociais", sendo possíveis as seguintes classificações: A1, A2, B1, B2, C, D e E ${ }^{17}$, em ordem decrescente de condição socioeconômica.

A coleta foi realizada por seis alunos do curso de Odontologia da Universidade de Fortaleza, e a equipe foi composta por três examinadoras e três anotadoras, que, previamente à coleta, foram submetidas a um processo de calibração ${ }^{15}$. Os dados coletados foram processados pelo software Excel $^{\circledR}$, conforme recomendação do Projeto SB-200015, e resultou numa boa concordância diagnóstica interexaminadora, cujos resultados foram de 0,90 para cárie de coroa, 0,92 para cárie de raiz, 0,91 para necessidade de prótese e 0,98 para necessidade de tratamento.

Os exames foram realizados com paciente sentado com a cabeça para trás, em cadeiras comuns dentro de seus domicílios, sob luz natural, utilizando apenas espelho bucal e sonda periodontal tipo OMS e de acordo com a técnica preconizada pela $\mathrm{OMS}^{16}$.

Os formulários foram revisados e um banco de dados foi criado usando o aplicativo EPIINFO e sua análise feita com o aplicativo estatístico STATA $9.0^{13}$.

A análise dos dados consistiu no cálculo das médias, e seus respectivos intervalos de confiança de 95\%, medianas, valores mínimos e máximos. Para testar hipóteses sobre a associação do uso e da necessidade de próteses (variáveis desfechos) com grupo etário, sexo, classe econômica e raça/etnia (variáveis independentes), foi calculado o qui-quadrado, com significância estatística $\mathrm{p}<0,05$.

O estudo não gerou riscos aos seus participantes e atendeu aos princípios da autonomia, beneficência, não maleficência e justiça, conforme disponibilizados na Resolução 196 de 1996 do Conselho Nacional de Saúde ${ }^{18}$. O projeto de pesquisa recebeu aprovação do Comitê de Ética em Pesquisa em Se- res Humanos da Unifor, Ceará, em 02 de junho de 2008, sob parecer 150/2008 e sob registro no CEP de 08-167 e CAAE 1550.0.000.037-08.

Todos os participantes assinaram o Termo de Consentimento Livre e Esclarecido. A privacidade e o sigilo das informações dos indivíduos foram rigorosamente respeitados por todos os pesquisadores e demais profissionais envolvidos nas etapas da pesquisa, da mesma forma que as informações obtidas a partir dos procedimentos específicos do projeto. Os nomes dos indivíduos participantes não aparecerão em publicações nem serão acessíveis a terceiros.

\section{Resultados}

No presente estudo, foram examinados 141 indivíduos, tendo 83 (59\%) entre 35 e 44 anos e 58 $(41 \%)$ mais de 65 anos. Ficou constatada a prevalência de cárie em $100 \%$ dessa população. Em relação ao número de dentes afetados por indivíduos, o valor mínimo encontrado foi igual a 1 e o máximo, igual a 32 .

Ao analisar o CPO-D, constatou-se que não houve diferença significativa na característica sexo, pois ambos apresentaram uma prevalência alta, sendo necessário ressaltar que pessoas do sexo feminino foram mais encontradas durante o estudo, representando aproximadamente $71 \%$ da amostra. Em relação ao grupo etário, foi possível observar um aumento do CPO-D com o aumento da idade, tendo a população acima de 65 anos apresentado média de 27,93 dentes com presença ou experiência de cárie dentária. Constatou-se uma semelhança no valor do CPO-D nas classes econômicas $\mathrm{C}$ e D, sendo os valores médios entre 21,63 e 22,42. Ainda, quanto à raça/cor, a maioria dos indivíduos entrevistados considerou-se negro, apresentando média de CPO-D de 21,75 (Tabela 1).

Tabela 1 - Número de examinados, média, intervalo de confiança, mediana, mínimo e máximo do CPOD, conforme sexo, grupo etário, classe econômica e raça/cor, Fortaleza-CE, 2010

\begin{tabular}{|c|c|c|c|c|c|c|}
\hline \multirow{2}{*}{ Características } & \multicolumn{6}{|c|}{ CPOD } \\
\hline & $\mathrm{n}$ & Média & IC de $95 \%$ & Mediana & Mín. & Máx. \\
\hline \multicolumn{7}{|l|}{ Sexo } \\
\hline Masculino & 41 & 21,51 & $20,19-22,83$ & 22 & 1 & 32 \\
\hline Feminino & 100 & 22,39 & $21,65-23,30$ & 22 & 1 & 32 \\
\hline \multicolumn{7}{|l|}{ Grupo etário } \\
\hline 35 a 44 anos & 83 & 18,08 & $17,36-18,8$ & 18 & 1 & 32 \\
\hline 65 a 74 anos & 58 & 27,93 & $27,24-28,62$ & 30 & 14 & 32 \\
\hline \multicolumn{7}{|l|}{ Classe econômica } \\
\hline $\mathrm{E}$ & 1 & 32 & - & 32 & 32 & 32 \\
\hline $\mathrm{D}$ & 52 & 22,42 & $21,38-23,46$ & 23 & 1 & 32 \\
\hline $\mathrm{C}$ & 83 & 21,63 & $20,76-22,5$ & 21 & 1 & 32 \\
\hline B2 & 5 & 25,4 & $22,23-28,57$ & 25 & 15 & 32 \\
\hline \multicolumn{7}{|l|}{ Raça/cor } \\
\hline Amarelo & 2 & 32,00 & - & 32 & 32 & 32 \\
\hline Branco & 5 & 23,20 & $17,41-28,99$ & 28 & 1 & 32 \\
\hline Indígena & 2 & 26,00 & $22,00-30,00$ & 26 & 22 & 30 \\
\hline Negro & 118 & 21,75 & $21,05-22,45$ & 22 & 1 & 32 \\
\hline Pardo & 14 & 23,00 & $21,01-24,99$ & 23,5 & 12 & 32 \\
\hline
\end{tabular}

Fonte: dados primários 
Não se obtiveram dados significantes quanto às classes econômicas $\mathrm{B} 2 \mathrm{e} \mathrm{E}$, nem quanto aos indivíduos que se consideraram amarelos e indígenas, visto não ter sido encontrado grande número de pessoas de tais grupos durante o estudo.

Referindo-se ao CPO-D passado, quanto ao sexo, tanto homens como mulheres apresentaram média semelhante, o que não ocorreu quanto ao grupo etário, em que a população idosa apresentou 12,72 elementos restaurados ou extraídos por cáries a mais do que a população adulta. Essa média de dentes perdidos e/ou obturados foi semelhante para as classes $\mathrm{C}$ e D e para pessoas da raça negra, sendo estas, respectivamente, $18,65,18,48$ e 18,35 (Tabela 2).

Tabela 2 - Número de examinados, média, intervalo de confiança, mediana, mínimo e máximo do CPOD passado, conforme sexo, grupo etário, classe econômica e raça/cor, Fortaleza-CE, 2010

\begin{tabular}{|c|c|c|c|c|c|c|}
\hline \multirow{2}{*}{ Características } & \multicolumn{6}{|c|}{ CPOD passado } \\
\hline & $\mathrm{n}$ & Média & IC de $95 \%$ & Mediana & Mín. & Máx. \\
\hline \multicolumn{7}{|l|}{ Sexo } \\
\hline Masculino & 41 & 18,41 & $16,94-19,88$ & 19 & 0 & 32 \\
\hline Feminino & 100 & 19,01 & $18,08-19,94$ & 17 & 0 & 32 \\
\hline \multicolumn{7}{|l|}{ Grupo etário } \\
\hline 35 a 44 anos & 83 & 13,60 & $12,86-14,34$ & 13 & 0 & 32 \\
\hline 65 a 74 anos & 58 & 26,32 & $25,39-27,25$ & 28,5 & 6 & 32 \\
\hline \multicolumn{7}{|l|}{ Classe econômica } \\
\hline E & 1 & 31,00 & - & 31 & 31 & 31 \\
\hline $\mathrm{D}$ & 52 & 18,48 & $17,19-19,77$ & 17 & 0 & 32 \\
\hline C & 83 & 18,65 & $17,61-19,69$ & 17 & 0 & 32 \\
\hline B2 & 5 & 23,20 & $19,04-27,36$ & 23 & 10 & 32 \\
\hline \multicolumn{7}{|l|}{ Raça/cor } \\
\hline Amarelo & 2 & 32,00 & - & 32 & 32 & 32 \\
\hline Branco & 5 & 18,60 & $12,15-25,05$ & 23 & 0 & 32 \\
\hline Indígena & 2 & 19,50 & $14,00-25,00$ & 19,5 & 14 & 25 \\
\hline Negro & 118 & 18,35 & $17,51-19,19$ & 17 & 0 & 32 \\
\hline Pardo & 14 & 21,00 & $18,71-23,29$ & 22 & 8 & 32 \\
\hline
\end{tabular}

Fonte: dados primários

Quando observada a atividade atual de cárie (CPO-D presente), comprova-se que a similaridade entre os sexos permanece, sendo a média de dentes cariados 3,09 para o sexo masculino e 3,38 para o feminino, semelhança que não ocorre no grupo etário, no qual os indivíduos adultos apresentaram uma média de 4,48 dentes cariados, sendo o máximo 15 , e os indivíduos idosos, uma média de 1,60, com um máximo de 21 unidades cariadas (Tabela 3 ).

Analisando o CPO-D presente quanto às variáveis classe econômica e raça/cor, indivíduos da clas- se D mostraram-se com a maior média de dentes cariados, apresentando um máximo de 21 unidades dentárias com atividade de cárie. O mesmo valor pode ser observado naqueles que se consideraram brancos, ressaltando que apenas 5 deste grupo foram encontrados no estudo. A população negra estudada, 118 indivíduos, que representa a maior parcela da amostra, apresentou uma média de dentes cariados menor que a das raças branca e indígena, com valores de $3,39,4,60$ e 6,50 , respectivamente (Tabela 3).

Tabela 3 - Número de examinados, média, intervalo de confiança, mediana, mínimo e máximo do CPOD presente, conforme sexo, grupo etário, classe econômica e raça/cor, Fortaleza-CE, 2010

\begin{tabular}{|c|c|c|c|c|c|c|}
\hline \multirow{2}{*}{ Características } & \multicolumn{6}{|c|}{ CPOD presente } \\
\hline & $\mathrm{n}$ & Média & IC de $95 \%$ & Mediana & Mín. & Máx. \\
\hline \multicolumn{7}{|l|}{ Sexo } \\
\hline Masculino & 41 & 3,09 & $2,57-3,61$ & 2 & 0 & 13 \\
\hline Feminino & 100 & 3,38 & $2,97-3,79$ & 2 & 0 & 21 \\
\hline \multicolumn{7}{|l|}{ Grupo etário } \\
\hline 35 a 44 anos & 83 & 4,48 & $4,05-4,91$ & 3 & 0 & 15 \\
\hline 65 a 74 anos & 58 & 1,60 & $1,17-2,03$ & 0 & 0 & 21 \\
\hline \multicolumn{7}{|l|}{ Classe econômica } \\
\hline$E$ & 1 & 1 & - & 1 & 1 & 1 \\
\hline $\mathrm{D}$ & 52 & 3,94 & $3,30-4,58$ & 2 & 0 & 21 \\
\hline $\mathrm{C}$ & 83 & 2,98 & $2,60-3,36$ & 2 & 0 & 15 \\
\hline B2 & 5 & 2,20 & $1,19-3,21$ & 2 & 0 & 5 \\
\hline \multicolumn{7}{|l|}{ Raça/cor } \\
\hline Amarelo & 2 & 0 & - & 0 & 0 & 0 \\
\hline Branco & 5 & 4,60 & $0,50-8,70$ & 1 & 0 & 21 \\
\hline Indígena & 2 & 6,50 & $5,00-8,00$ & 6,5 & 5 & 8 \\
\hline Negro & 118 & 3,39 & $3,05-3,73$ & 2 & 0 & 15 \\
\hline Pardo & 14 & 2,00 & $1,38-2,62$ & 1.5 & 0 & 6 \\
\hline
\end{tabular}

Fonte: dados primários 
Na amostra, $39(27,7 \%)$ dos indivíduos estudados apresentaram baixo CPOD, enquanto $102(72,4 \%)$ tiveram alto CPOD. As características de exposição (sexo, grupo etário, raça/cor e classe econômica) não evidenciaram relação causal de risco para os indivíduos que apresentaram um alto CPOD.

No que se refere à situação protética dentro da população, as mulheres usam mais prótese superior quando comparadas aos homens $(\mathrm{p}=0,016)$ (Tabela 4). Estes, por sua vez, têm maior necessidade de utilização dessa prótese $(\mathrm{p}=0,008)$ (Tabela 5). Na análise entre grupos etários, tanto no uso de pró- tese superior $(p=0,001)$ quanto no de inferior $(p=$ 0,000 ), a população idosa teve maiores percentuais, $63,79 \%$ e $32,76 \%$ (Tabela 4). A necessidade de prótese superior entre os adultos foi bem alta, $53,01 \%$, sendo a maior necessidade advinda de uma combinação entre prótese removível e fixa $(p=0,049)$ (Tabela 5). A classe econômica e a raça/cor não evidenciaram estatisticamente a sua relação com o uso e a necessidade de prótese superior e inferior, tendo sido relacionadas somente com o maior uso de prótese inferior entre os pardos $(28,57 \%-p=0,012)$ (Tabelas 4 e 5).

Tabela 4 - Número de examinados, porcentagem, intervalo de confiança e valor de p, para uso de prótese, conforme sexo, grupo etário, classe econômica e raça/cor, Fortaleza-CE, 2010

\begin{tabular}{|c|c|c|c|c|c|c|c|}
\hline \multirow{2}{*}{ Características } & \multirow{2}{*}{$n$} & \multicolumn{3}{|c|}{ Usa prótese superior } & \multicolumn{3}{|c|}{ Usa prótese inferior } \\
\hline & & $\%$ & IC de $95 \%$ & Valor de $p$ & $\%$ & IC de $95 \%$ & Valor de $p$ \\
\hline \multicolumn{8}{|l|}{ Sexo } \\
\hline Masculino & 41 & 31,71 & $19,39-47,27$ & 0,016 & 12,20 & $5,17-26,14$ & 0,397 \\
\hline Feminino & 100 & 54,00 & $44,20-63,50$ & & 18,00 & $11,64-26,7$ & \\
\hline \multicolumn{8}{|l|}{ Grupo Etário } \\
\hline $35-44$ anos & 83 & 36,14 & $26,56-46,97$ & 0,001 & 4,82 & $1,82-12,15$ & 0,000 \\
\hline mais de 65 anos & 58 & 63,79 & $50,77-75,06$ & & 32,76 & $21,97-45,7$ & \\
\hline \multicolumn{8}{|l|}{ Classe econômica } \\
\hline$E$ & 1 & 100,00 & - & 0,066 & 0,00 & - & 0,347 \\
\hline $\mathrm{D}$ & 52 & 48,08 & $34,96-61,47$ & & 11,54 & $5,28-23,39$ & \\
\hline $\mathrm{C}$ & 83 & 43,37 & $33,17-54,18$ & & 18,07 & $11,20-27,84$ & \\
\hline B2 & 5 & 100,00 & - & & 40,00 & $10,02-79,96$ & \\
\hline \multicolumn{8}{|l|}{ Raça/cor } \\
\hline Amarelo & 2 & 100,00 & - & 0,608 & 100,00 & - & 0,012 \\
\hline Branco & 5 & 60,00 & $20,04-89,98$ & & 20,00 & $2,72-69,10$ & \\
\hline Negro & 118 & 45,76 & $37,00-54,79$ & & 13,56 & $8,48-20,99$ & \\
\hline Pardo & 14 & 50,00 & $25,97-74,03$ & & 28,57 & $11,15-56,05$ & \\
\hline Indígena & 2 & 50,00 & $5,89-94,11$ & & 0,00 & - & \\
\hline
\end{tabular}

Fonte: dados primários

Tabela 5 - Número de examinados, porcentagem, intervalo de confiança e valor de p, para necessidade de prótese, conforme sexo, grupo etário, classe econômica e raça/cor, Fortaleza-CE, 2010

\begin{tabular}{|c|c|c|c|c|c|c|c|}
\hline \multirow{2}{*}{ Características } & \multirow{2}{*}{$\mathrm{n}$} & \multicolumn{3}{|c|}{ Necessidade de prótese superior } & \multicolumn{3}{|c|}{ Necessidade de prótese inferior } \\
\hline & & $\%$ & IC de $95 \%$ & Valor de $p$ & $\%$ & IC de $95 \%$ & Valor de $\mathrm{p}$ \\
\hline \multicolumn{8}{|l|}{ Sexo } \\
\hline Masculino & 41 & 63,41 & $47,87-76,59$ & 0,008 & 78,05 & $62,93-88,16$ & 0,613 \\
\hline Feminino & 100 & 39,00 & $29,96-48,86$ & & 74,00 & $64,55-81,65$ & \\
\hline \multicolumn{8}{|l|}{ Grupo etário } \\
\hline 35 a 44 anos & 83 & 53,01 & $42,30-63,45$ & 0,049 & 80,72 & $70,82-87,84$ & 0,068 \\
\hline mais de 65 anos & 58 & 36,21 & $24,94-49,23$ & & 67,24 & $54,26-78,03$ & \\
\hline \multicolumn{8}{|l|}{ Classe econômica } \\
\hline $\mathrm{E}$ & 1 & 100,00 & - & 0,117 & 100,00 & - & 0,694 \\
\hline $\mathrm{D}$ & 52 & 44,23 & $31,45-57,82$ & & 44,23 & $31,45-57,82$ & \\
\hline $\mathrm{C}$ & 83 & 49,40 & $38,83-60,02$ & & 49,40 & $38,83-60,02$ & \\
\hline B2 & 5 & 0,00 & - & & 0,00 & - & \\
\hline \multicolumn{8}{|l|}{ Raça/cor } \\
\hline Amarelo & 2 & 0,00 & - & 0,496 & 0,00 & - & 0,069 \\
\hline Branco & 5 & 20,00 & $2,72-69,10$ & & 60,00 & $20,04-89,98$ & \\
\hline Negro & 118 & 48,31 & $39,44-57,28$ & & 77,97 & $69,60-84,54$ & \\
\hline Pardo & 14 & 42,86 & $20,65-68,37$ & & 64,29 & $37,63-84,30$ & \\
\hline Indígena & 2 & 50,00 & $5,89-94,11$ & & 100,00 & - & \\
\hline
\end{tabular}

Fonte: dados primários 
Dentre os examinados que necessitam usar ou usam próteses, analisada a situação individual por meio de cada tipo de prótese, foi observado que 38 indivíduos usam prótese total superior e 18 usam a inferior. Constatou-se, ainda, que 10 indivíduos necessitam de completa reabilitação superior e 12 , de inferior.

\section{Discussão}

Com base nos dados existentes na literatura, sabe-se que as condições de saúde bucal da população adulta e idosa no Brasil são precárias, o que decorre, principalmente, do antigo padrão de serviços em saúde bucal, aos quais existia dificuldade de acesso, e, quando essa barreira era superada, o usuário deparava-se com extração dentária em massa como solução mais prática e econômica para a resolução de seus problemas ${ }^{19,20}$. Isso pode ser confirmado no presente estudo, no qual a experiência de cárie deu-se em todos os indivíduos da pesquisa. A proposta de estudar-se as condições bucais desses grupos é adequada à necessidade de fundamentar ações concretas de intervenção que visem a melhorar os padrões de saúde bucal da população.

Dentro da amostra do estudo, houve recusas dos participantes, impossibilidade de localizá-los ou de localizar seus domicílios, além de domicílios demolidos ou fechados. O alto percentual de domicílios não visitados $(23,04 \%)$ deveu-se tanto aos endereços incompletos como à rapidez dos deslocamentos, uma vez que a concentração urbana, aliada à informalidade e à precariedade no mercado de trabalho, auxilia na aceleração da mobilidade e no colapso das formas de provisão de moradia. Com efeito, a concentração de renda nas áreas mais centrais das metrópoles mantém uma forte pressão pela ocupação nesses pontos como condição de acesso à renda para os segmentos de trabalhadores subemprega$\operatorname{dos}^{21}$.

Dentre os indivíduos estudados, na distribuição quanto ao sexo, observou-se que o número de mulheres encontradas nos domicílios predominava em relação ao dos homens, corroborando os estudos de Ferreira Filho et al. ${ }^{22}$ (1996) e Andrade et al. ${ }^{23}$ (2006). É importante ressaltar que as visitas foram realizadas no período da manhã, quando a maioria dos homens cadastrados na pesquisa estava no trabalho. Assim, foram encontradas mais mulheres, pelo fato de estarem mais propícias ao trabalho doméstico e ao cuidado dos filhos.

Quanto ao grupo etário, verifica-se que a perda e/ou restauração dos elementos dentários é maior na população idosa, em decorrência da associação entre a falta de ações preventivas e educativas com os maiores riscos desse grupo, devido a má higiene bucal, ingestão excessiva de açúcar, menor acesso ao flúor, produção salivar diminuída, tabagismo, alcoolismo, quadros de demência e alterações sistêmicas ${ }^{24}$. Esse cenário torna-se ainda mais grave quando o CPO-D passado $(26,32)$ revela-se maior que o encontrado para a região Nordeste durante o levantamento epidemiológico realizado em 2003 para indivíduos do mesmo grupo etário $(25,54)^{25}$. Dentro da experiência de cárie, o componente perdido $(\mathrm{P})$ é mais observado, o que se repete em diversos estudos $^{26,27}$, explicando o maior uso de prótese pelo idoso.

Indivíduos adultos, por sua vez, apresentam uma atividade presente de cárie maior que os idosos, com o CPO-D presente $(4,48)$, valor maior que o encontrado no Projeto SB Brasil $2003^{25}$ para a região Nordeste $(2,86)$, podendo relacionar-se com o padrão de uso dos serviços odontológicos, como relatado no estudo de Gilbert et al. ${ }^{28}$ (1997) e Matos et al. ${ }^{29}$ (2001). Isso se deve ao fato de que pessoas com uma renda financeira menor têm maior dificuldade de acesso aos serviços odontológicos, gerando maiores problemas bucais. Além disso, uma baixa posição socioeconômica está associada a baixos níveis de escolaridade, precárias condições de habitação e trabalho e falta de interesse individual pela procura regular dos serviços, todos esses sendo fatores não clínicos responsáveis por potencializar o risco de cárie $^{30,31}$.

As médias de CPOD passado foram apresentadas, em todas as características, superiores às médias do CPOD presente, afirmando a presença de uma odontologia mutiladora nas populações adulta e idosa do presente estudo. A perda dos dentes é o reflexo das desigualdades sociais, sendo a reabilitação protética uma forma de minimizar os danos estético, mastigatório, fonético e até mesmo psicossocial. Quanto ao uso de prótese, verifica-se a maior ocorrência no sexo feminino, podendo ser devido à menor disponibilidade dos homens em procurar por serviços odontológicos.

$\mathrm{O}$ uso de prótese é predominantemente maior na arcada superior, somando $47,5 \%$, contra $16,3 \%$ que fazem uso de prótese inferior. Isso não se repete quanto à necessidade, visto que a arcada inferior mostrou-se a mais necessitada de algum tipo de reabilitação protética, pois, ao analisar-se sexo, grupo etário, classe econômica e raça/cor, verificou-se que $75,1 \%$ das pessoas necessitavam de reabilitação inferior, enquanto $46 \%$ necessitavam de superior.

Dentre os tipos de prótese, a total é a que representa a completa perda das unidades dentárias. A população idosa apresentou-se mais necessitada de seu uso, diferentemente dos adultos, entre a qual a mais necessária foi uma combinação de próteses, achado que se repete em toda a população brasileira $^{25}$. O uso de prótese total superior foi $14,18 \%$ maior que o uso da inferior, também verificado em estudo feito com idosos institucionalizados em Fortaleza $^{26}$ e em visitas domiciliares no município de Biguaçu $^{20}$. Quanto à necessidade, a prótese total 
inferior foi $1,42 \%$ mais necessária que a superior. Observa-se que, apesar da necessidade de reabilitação inferior ser maior, a superior é mais prevalente, o que ocorre devido à dificuldade de adaptação e ao desconforto no arco inferior, além de razões estéticas e econômicas ${ }^{20}$.

Verificou-se que a necessidade de algum tipo de reabilitação protética no estudo foi, para a arcada superior, de $53,01 \%$ para adultos e de $36,21 \%$ para idosos e, para a arcada inferior, de $80,72 \%$ para adultos e de 67,24\% para idosos, apresentando um padrão de necessidade maior que a encontrada para a população brasileira, a qual foi, para a arcada superior, de $35,83 \%$ em indivíduos adultos e de $32,40 \%$ em idosos, e, para a inferior, de $70,99 \%$ em adultos e de 50,06\% em idosos ${ }^{25}$. Isso demonstra a pouca resolutividade dos serviços protéticos, valendo ressaltar que estes foram oferecidos pelos Centros de Especialidades Odontológicas somente a partir de 2003, começando a fazer parte das opções de tratamento ${ }^{32}$.

\section{Conclusão}

Assim, por meio deste estudo, podemos concluir que sexo, classe econômica e raça/cor não influenciaram o CPOD, o CPOD presente e o CPOD passado; todavia, os idosos possuem maior CPOD e CPOD passado, enquanto os adultos possuem maior CPOD presente. Conclui-se, também, que as mulheres usam mais próteses superiores; idosos usam mais próteses superiores/inferiores e pardos, inferiores. Homens e adultos, por sua vez, têm mais necessidade de utilizar próteses superiores.

Existe, ainda, a real necessidade de reparar os danos causados pela odontologia mutiladora do passado, reabilitando o paciente com serviços de prótese resolutivos e reintegrando-o ao convívio social, além de prevenir novas perdas dentárias por meio de políticas públicas de saúde, com o intuito de modificar o atual retrato bucal do país, que reflete as desigualdades existentes na sociedade brasileira. Assim, será realmente possível afirmar que somos um "Brasil sorridente"!

\section{Abstract}

Objectives: this research aims to study the relationship between socioeconomic and demographic factors, and dental caries and their outcomes in adult and elderly residents of a Northeastern community. Methods: questionnaires were applied to characterize household and socioeconomic conditions. Oral examinations were held in 141 subjects, 83 (59\%) of them aged 35 to 44, and 58 (41\%) of the over 65 years old. Data analysis consisted on the calculation of means, confidence intervals of $95 \%$, and median, minimum and maximum values. To test hypotheses about the association of the use and need of prostheses with age group, gender, economic status, and race/ethnicity, the chi-square test was applied. The statistical significance level of 5\% was used. Results: in the analysis of DMFT, there was no significant difference regarding genders, since both presented high prevalence. The black population studied presented less decayed teeth than white and Indian races in average, with values of 3.39, 4.60, and 6.50, respectively. Women more often use upper prosthesis when compared to men $(p=0.016)$. They, in turn, have increased need of that prosthesis $(p=0.008)$. Conclusion: thus, through this study we can conclude that gender, economic status, and race/color did not affect the DMFT.

Descriptors: Oral health. Health surveys. Dental caries. Social conditions.

\section{Referências}

1. Boing AF, Peres MA, Kovaleski DF, Zange SE, Antunes JLF. Estratificação sócio-econômica em estudos epidemiológicos de cárie dentária e doenças periodontais: características da produção na década de 90. Cad Saúde Pública 2005; 3:673-8.

2. COMMISSION ON SOCIAL DETERMINANTS OF HEALTH (CSDH). A conceptual framework for action on social determinants of health [2010 Nov 19]. Disponível em URL: www.determinantes.fiocruz.br.

3. Buss PM, Pellegrini Filho A. A saúde e seus determinantes sociais. PHYSIS: Rev Saúde Coletiva 2007; 1:77-93.

4. Loretto NRM, Seixas ZA, Jardim MC, Brito RL. Cárie dentária no Brasil: alguns aspectos sociais, políticos e econômicos. Rev ABO Nac 2000; 1:45-9.

5. Abreu MHNG, Modena CM, Pordeus IA. Determinantes sociais na epidemiologia da cárie dentária. Rev Odonto Ciências 2004; 46:360-6.

6. Locker D. Deprivation and oral health: a review. Community Dent Oral Epidemiol 2000; 28:161-9.

7. Barbato PR, Nagano HCM, Zanchet FN, Boing AF, Peres MA. Perdas dentárias e fatores sociais, demográficos e de serviços associados em adultos brasileiros: uma análise dos dados do Estudo Epidemiológico Nacional (Projeto SB Brasil 2002-2003). Cad Saúde Pública 2007; 8:1803-1314 .

8. Gesser HC, Peres MA, Marcenes W. Condições gengivais e periodontais associadas a fatores socioeconômicos. Rev Saúde Pública 2001; 3:289-93.

9. Moreira RS, Nico LS, Tomita NE, Ruiz T. A saúde bucal do idoso brasileiro: revisão sistemática sobre o quadro epidemiológico e acesso aos serviços de saúde bucal. Cad Saúde Pública 2005; 6:1665-75.

10. Fure S, Zickert I. Incidence of tooth loss and dental caries in 60, 70 and 80 years old Swedish individuals. Community Den Oral Epidemiol 1997; 2:137-42.

11. Narvai PC, Frazão P, Roncalli AG, Antunes JLF. Cárie dentária no Brasil: declínio, polarização, iniquidade e exclusão social. Rev Panam Salud Publica 2006; 6:385-93.

12. Moura KS, Bessa OAAC, Nuto SAS, Sá HLC, Veras FMF, Braga JU. Projeto Coorte Dendê: diagnóstico demográfico e condições de moradia de uma comunidade de baixa renda em Fortaleza-Ceará. RBPS 2010; 1:18-24. 
13. Estados Unidos. StataCorp. STATA Statistical Software. V9.0. Release 9.0 ed. College Station, Texas 77845 USA: Stat Corporation: 2007.

14. Martins MCA, Landim JR, Freire Neta MCA. Projeto Coorte Dendê: caracterização domiciliar. In: $17^{\circ}$ Encontro de Iniciação à Pesquisa UNIFOR; 2011 Out 17-21; Fortaleza. Anais. Fortaleza: Gráfica da Universidade de Fortaleza; 2011. p. 1-4.

15. Brasil. Ministério da Saúde. Secretaria de Políticas da Saúde. Departamento de Atenção Básica. Área Técnica de Saúde Bucal. Projeto SB 2000: condição de saúde bucal da população brasileira no ano de 2000. Manual do examinador. Brasília: Ministério da Saúde; 2001.

16. Organização Mundial da Saúde (OMS). Levantamentos básicos em saúde bucal, 4. ed. São Paulo: Ed. Santos; 1999.

17. Associação Nacional de Empresas de Pesquisa. São Paulo: critério de classificação econômica Brasil [citado 2010 Maio 27]. Disponível em URL: http://www.datavale-sp.com.br/ cceb.

18. Conselho Nacional de Saúde (BR). Resolução n. 196/96. Dispõe sobre diretrizes e normas regulamentadoras de pesquisas envolvendo seres humanos. Brasília: Conselho Nacional de Saúde; 1996.

19. Vargas AMD, Paixão HH. Perda dentária e seu significado na qualidade de vida de adultos usuários de serviço público de saúde bucal do Centro de Saúde Boa Vista, Belo Horizonte. Ciência \& Saúde Coletiva 2005; 4:1015-24.

20. Colussi CF, Freitas SFT, Calvo MCM. Perfil epidemiológico da cárie e do uso e necessidade de prótese na população idosa de Biguaçu, Santa Catarina. Rev Bras Epidemiol 2004; 1:8897.

21. Ribeiro LCQ. Dinâmica socioterritorial das metrópoles brasileiras: dispersão e concentração. Instituto de Pesquisa Econômica e Aplicada - IPEA. Políticas Sociais: Acompanhamento e Análise 2006; 12:221-9.

22. Ferreira FJS, Cavalcanti MT, Cadilhe GR, Vilaça AT, Avancini E, Machado HO et al. Avaliação de qualidade de serviços de saúde mental no município de Niterói-RJ: a satisfação do usuário como critério avaliador. J Bras Psiquiat 1996; 7:393402.

23. Andrade KLC, Ferreira EF. Avaliação da inserção da odontologia no Programa Saúde da Família de Pompeu (MG): a satisfação do usuário. Ciência e Saúde Coletiva 2006; 1:12330.

24. Campostrini EP, Ferreira EF, Rocha FL. Condições da saúde bucal do idoso brasileiro. Arquivos em Odontologia 2007; 2:48-56.

25. Brasil. Ministério da Saúde. Secretaria de Políticas de Saúde. Departamento de Atenção Básica, Coordenação Nacional de Saúde Bucal. Projeto SB Brasil 2003. Condições de Saúde Bucal da População Brasileira: 2002-2003. Resultados principais. Brasília: Ministério da Saúde; 2004.

26. Gaião LR, Almeida MEL, Heukelbach J. Perfil epidemiológico da cárie dentária, doença periodontal, uso e necessidade de prótese em idosos residentes em uma instituição na cidade de Fortaleza, Ceará. Rev Bras Epidemiol 2005; 3:316-23.

27. Colussi CF, Freitas SFT. Aspectos epidemiológicos da saúde bucal do idoso no Brasil. Cad Saúde Pública 2002; 5: 1313-20.
28. Gilbert HG, Duncan RP, Heft MW, Coward RT. Dental health attitudes among dentate black and white adults. Medical Care 1997; 35:255-71.

29. Matos DL, Lima-Costa MFF, Guerra HL, Marcenes W. Projeto Bambuí: Estudo de base populacional dos fatores associados com o uso regular de serviços odontológicos em adultos. Cad Saúde Pública 2001; 3:661-8.

30. Morando V, Miranda L, Klein ALL, Lima FAP. Levantamento Epidemiológico e Doença Cárie. Arq Ciênc Saúde Unipar 2003; Supl:33-5.

31. Camargo MBJ, Dumith SC, Barros AJD. Uso regular de serviços odontológicos entre adultos: padrões de utilização e tipos de serviços. Cad Saúde Pública 2009; 9: 1894-1906.

32. Brasil. Ministério da Saúde. Secretaria de Atenção à Saúde. Departamento de Atenção Básica. Coordenação Nacional de Saúde Bucal. Diretrizes da política nacional de saúde bucal. Brasília: Ministério da Saúde; 2004.

\section{Endereço para correspondência:}

Mychele Cavalcante de Andrade Martins Rua Gerôncio Brígido Neto, 60/301 Bairro: Praia do Futuro CEP 60.182-335 Fortaleza-CE Fone: (85) 88940603

E-mail: mychele_andrade@hotmail.com

Recebido: 04/04/2013. Aceito: 02/06/2013. 